\title{
Method for Analysis of Nanoparticle Hemolytic Properties In Vitro
}

\author{
Marina A. Dobrovolskaia ${ }^{*}$, Jeffrey D. Clogston, Barry W. Neun, Jennifer B. Hall, Anil K. \\ Patri, and Scott E. McNeil \\ Nanotechnology Characterization Laboratory, Advanced Technology Program, SAIC-Frederick \\ Inc., NCl-Frederick, Frederick, MD 21702
}

\begin{abstract}
Hemolysis (destruction of red blood cells) in vivo can lead to anemia, jaundice and other pathological conditions, therefore the hemolytic potential of all intravenously administered pharmaceuticals must be evaluated. Nanotechnology-derived devices and drug carriers are emerging as alternatives to conventional small-molecule drugs, and in vitro evaluation of their biocompatibility with blood components is a necessary part of early preclinical development. The small size and unique physicochemical properties of nanoparticles may cause their interactions with erythrocytes to differ from those observed for conventional pharmaceuticals, and may also cause interference with standardized in vitro tests. Separating true hemolytic responses from the false-positive or falsenegative results caused by particle interference is important for correct interpretation of these tests. Here we describe validation of an in vitro assay for the analysis of nanoparticle hemolytic properties, and discuss observed nano-interferences with the assay. We propose alternative methods to avoid misleading results from nanoparticles, and discuss the potential relevance of nanoparticle in vitro hemolytic properties to in vivo systems.
\end{abstract}

\section{Keywords}

nanoparticles; hemolysis; in vitro assay; validation; interference; false-positive; false-negative

\section{Introduction}

Nanoparticles are a relatively new class of biomedical products. Their potential use in medical devices or as drug carriers offers opportunities for novel therapy of complex disorders such as cancer, inflammatory, and neurodegenerative diseases 1,2 . As with any device or pharmaceutical,nanoparticles intended for biomedical application must be subject to biocompatibility testing before regulatory approval for administration to patients. Although numerous efforts are underway to define the critical parameters which must be addressed during preclinical evaluation of nanomaterials engineered for use in medicine ${ }^{3-6}$, few, if any, harmonized protocols for testing nanoparticle biocompatibility are currently available.

Determination of hemolytic properties is one of the most common tests in studies of nanoparticle interaction with blood components $7-25$. Interpreting the results of these studies is complicated due to variability in experimental approaches and a lack of universally accepted criteria for determination of the test-result validity. Most in vitro studies of particle-induced hemolysis $7-14,17-20,22-25$ evaluate the percent hemolysis by spectrophotometriclly detecting plasma free hemoglobin derivatives after incubating the particles with blood and then

*Correspondence and requests for materials should be addressed to M.A.D. (marina@ mail.nih.gov)., Nanotechnology Characterization Lab, SAIC-Frederick Inc., NCI-Frederick, 1050 Boyles St., Bldg. 469, Frederick MD, 21702, phone: (301)-846-6939, fax:

(301)-846-6399, e-mail: ncl@mail.nih.gov. 
separating undamaged cells by centrifugation. The incubation time, wavelength at which hemoglobin is quantified, and blood conditions (e.g. use of purified erythrocytes rather than whole blood, and inclusion of various anti-coagulants) vary significantly from one study to another. In addition to these variables, differences in relative centrifugal force, blood storage time and conditions, blood sources (human or rabbit) can further complicate meaningful comparison of the results from disparate studies.

Here, we integrate aspects of several methods for analysis of hemolytic properties into a 96well plate assay, and optimized this assay for testing nanoparticles. Our assay leverages an existing standard practice (ASTM F-756-00) for analysis of hemolytic properties of medical devices 26 . We scaled this standard practice to a 96-well plate format assay and conducted a one month validation aimed at determining its reproducibility, precision, and accuracy, as well as qualification of negative and positive nanoparticle-relevant controls. We refer to this initial phase of the validation as pre-study validation. We subsequently used our assay to analyze various types of nanomaterials including polymers, gold nanoshells, nanoliposomes, nanoemulsions, fullerene derivatives, gold colloids, and dendrimers. We refer to this second phase as in-study validation. The second phase was conducted over a two year period, and included identification and resolution of nanoparticle interference with the assay, in addition to evaluation of reproducibility, precision, accuracy and control qualification. In this report we present assay performance from both phases (pre-and in-study) of validation, and discuss the observed interference with the assay which we ascribe to uniquely "nano" interactions. We also provide practical solutions for overcoming these interferences, and define critical parameters which must be monitored to avoid spurious (false-positive or false-negative) results.

\section{Experimental}

\subsection{Assay Development and Validation}

The assay presented here is an adaptation of existing standard F-756-00 26 , which is based on colorimetric detection of red-colored cyanmethemoglobin in solution. In this assay, analyte particles are incubated in blood, hemoglobin released by damaged cells is oxidized to methemoglobin by ferricyanide in the presence of bicarbonate, then cyanide converts the methemoglobin to cyanmethemoglobin. The undamaged erythrocytes are removed by centrifugation, and the amount of cyanmethemoglobin in the supernatant is measured by spectrophotometry at its absorbance maximum wavelength, $540 \mathrm{~nm}$. This measured absorbance is compared to a standard curve to determine the concentration of hemoglobin in the supernatant, and this hemoglobin concentration is compared to that in the supernatant of a blood sample not treated with nanoparticles to obtain the percentage particle-induced hemolysis (referred to as percent hemolysis). The standard curve is created from a linear fit of several absorbance measurements made at $540 \mathrm{~nm}$ on a hemoglobin standard sample (treated with ferricyanide and bicarbonate) over a range of hemoglobin concentrations from 0.025 to $80 \mathrm{mg} / \mathrm{mL}$ (we refer to these samples as "calibration standards").

The precision of the measured hemoglobin concentrations (determined as percent coefficient variation, \% CV) and accuracy (determined as percent difference from theoretical, PDFT), with the theoretical concentration corresponding to the value of the standard curve are calculated for each sample over all assay runs. $[h]$ is the mean measured hemoglobin concentration for a particular sample over all runs, and $\% \mathrm{CV}$ is the percentage of the mean of the standard deviation $(\% \mathrm{CV}=100 \times \mathrm{SD} /[h])$ and $\% \mathrm{DFT}$ is the percent difference of the mean concentration from the theoretical concentration $\left(\mathrm{PDFT}=100 \times\left(\frac{[h]-[h]_{\text {theory }}}{[h]_{\text {theory }}}\right)\right.$. In addition the calibration standards, the assay includes measurement on hemoglobin standard samples (treated with 
ferricyanide and bicarbonate) with known concentrations, we refer to these samples as "quality controls".

The experimental procedure described in the ASTM standard was modified by: 1) scale to a 96-well plate format, 2) introduction of nanoparticle-relevant controls, and 3) modification of acceptance criteria to reflect ICHS6 requirements for bioanalytical method validation 27 . The results of this assay (expressed as percentage hemolysis with respect to negative control) are used to evaluate the acute in vitro hemolytic properties of nanoparticles. The detailed experimental procedure is available at the NCL website 28 and in the supplementary materials section.

\subsection{Reagents}

Cyanmethemoglobin $(\mathrm{CMH})$ reagent and Hemoglobin standard were purchased from StanBio. $\mathrm{Ca}^{2+} / \mathrm{Mg}^{2+}$ free DPBS, Polyethylene glycol (av. MW 8 000), and Poly-L-Lysine (PLL) hydrobromide (MW 150000 - 300 000) were from Sigma-Aldrich. Polystyrene nanoparticles with size of 20,50 and 80nm were purchased from Duke Scientific Corporation. Colloidal gold nanoparticles with size of 5, 10, 20, 30, 40 and 50nm were from TedPella Inc. G5 and G6 PAMAM dendrimers with amine, carboxy and hydroxyl surface were Dendritic Nanotechnologies Inc. Nanoliposomes were kindly provided by Dr. Mark Kester (Pennsylvania State University). Nanoemulsions were kindly provided by Dr.Mansoor Amiji (Northeastern University). Triazine dendrimers and water-soluble fullerene derivatives (C3) were kindly provided by Dr. Eric Simanek (Texas A\&M University) and C60 Inc., respectively. Abraxane, Propofol and Doxil were purchased through NIH pharmacy.

\subsection{Research Donor Blood}

Healthy volunteer blood specimens were drawn under NCI-Frederick Protocol OH99-C-N046. Blood was collected in BD vacutainer tubes containing Lithium heparin (hemolsyis test) or sodium citrate (platelet aggregation test) as anticoagulant. Specimens from at least three donors were pooled.

\section{Results and Discussion}

\section{1. Assay validation}

The calibration standards and quality controls for both pre-study and in-study validation were prepared as described in the experimental section. Measurement of assay precision (determined as percent coefficient variation $(\% \mathrm{CV})$ ) and accuracy (determined as percent difference from theoretical (PDFT), with the theoretical value corresponding to the value of the standard curve) were calculated from seven and twelve standard curves for pre-study and in-study validation, respectively. The results of the first (pre-study) phase are presented in Table 1A. The results of the second (in-study) phase are summarized in Table 1B. In both phases we have observed high precision and accuracy, in that both the \%CV and PDFT are less than 10\%. The lowest calibration standard quantification was slightly less accurate (PDFT=-14.3\%) in the second phase (Table 1B). These values are within the limits suggested for analytical assays by the US FDA $^{29}$ and those used in industry in support of pharmaceutical development 27,30 .

To determine intra-assay (i.e. within one run) precision and accuracy, quality control hemoglobin standard samples were analyzed six times in one pre-study validation run. The results are presented in Table 1B. Both precision and accuracy in this test were high, as \%CV and PDFT were below $10 \%$.

Inter-assay precision and accuracy were determined by analysis of three quality control hemoglobin standard samples, each analyzed the pre-study and in-study validation, as 
described above. The quality control samples were prepared from a hemoglobin standard of a known concentration of $80.0 \mathrm{mg} / \mathrm{mL}$. Theoretical (calculated) concentrations of the quality control samples were as follows: 0.0625 (low), 0.125 (mid) and 0.625 (high) $\mathrm{mg} / \mathrm{mL}$. The results for pre-study validation are presented in Table $1 \mathrm{~A}$. The results of in-study validation for quality controls are summarized in Table 1B. The data demonstrated high precision and accuracy (\%CV and PDFT <10\%), with the exception of one low QC level in the in-study validation, for which variability between runs $(\% \mathrm{CV})$ was $12 \%$. This low precision is still within the limits suggested by the FDA, and was for the lowest concentration calibration standard, which is most prone to variable absorbance readings.

This assay validation demonstrates that our 96-well format assay for quantitative determination of hemoglobin is reproducible and robust. Although there is no formal recommendation to the cut-off limit for precision and accuracy of the hemoglobin-specific assay, the obtained values are within requirements described for small molecules, i.e. within $15 \% 27,29$.

\subsection{Selection and Qualification of Positive and Negative Controls}

An important aspect of an accurate evaluation of a material's hemolytic properties is the use of relevant positive and negative controls. ASTM standard F-756-00 was developed for medical devices, and uses controls which are impractical for the evaluation of nanoparticles. Since the small size and unique physicochemical properties of nanoparticles may cause a variety of nanoparticle-specific interferences, initially, we aimed to include controls which were nanoparticles themselves. Polyethyleneglycol (PEG) solution was chosen as a negative control since this polymer is frequently attached to nanoparticles to increase compatibility with blood components 1,31 . Selection of the positive control was more challenging; originally we chose cationic polystyrene nanoparticles with nominal sizes of 20, 50 and $80 \mathrm{~nm}$. Initially, high hemolytic activity was observed with each of these formulations (data not shown). However, when the surfactant/detergent Triton X-100 is removed from the polystyrene particle solution by dialysis prior to the assay, the particles adsorb plasma-free hemoglobin, aggregate and are removed along with undamaged cells during centrifugation. Triton X-100 is known to be hemolytic $^{10}$, and this was the reason for excluding polystyrene nanoparticles from positive control qualification. Another polymer which may produce a hemolytic response due to its high positive charge is Poly-L-lysine (PLL). Initially, this polymer, when tested in our assay, produced percent hemolysis greater than 8\%, which according to the ASTM standard F756 qualifies this material as a positive control. Inter-assay performance of the positive (PLL) and negative (PEG) control samples was evaluated during pre-study validation. We compared the percent hemolysis calculated for each control sample from six validation runs. The results are presented in Table 2A. To compare intra-assay performance, the positive and the negative control samples were analyzed six times in one validation run. The results are also presented in Table 2A.

Further studies of PLL in 96-well format assay revealed a high degree of inter-lot variability, depending on the lot of PLL and storage time, the percent hemolysis observed with this material varied from 5 to $70 \%$ (Figure 1). Although precision and accuracy of PLL in pre-study validation conducted using the same lot of polymer met the acceptance limits (i.e. \% CV and PDFT $<15 \%$, Table 2A), the high degree of inter-lot variability (Figure 1) disqualified this material from further use as positive control in this assay.

The only material, which produced hemolysis consistently over the entire two year period was Triton-X 100 (Table 2B). This is consistent with previously published studies ${ }^{12}$, although other studies have used distilled water as a positive control ${ }^{14}$. As with the calibration standards and quality controls, the variability in percent hemolysis (\%CV) induced by Triton-X 100 and PEG in in-study validation has met the industrial requirement of being within $15 \% 27,30$. 
Other parameters evaluated during assay validation included testing the effects of using freshly drawn blood versus stored blood, various blood incubation times with the test-materials, and different types of mixing during incubation (rotation versus shaking every 30 minutes). The results of these tests indicated that freshly-drawn blood can be stored up to 24 hours at $2-8^{\circ} \mathrm{C}$ with no appreciable effects on assay performance; prolonged storage (36 and $48 \mathrm{~h}$ ) resulted in gradual increase in plasma free hemoglobin (PFH), which disqualified blood samples with PHF levels above $1 \mathrm{mg} / \mathrm{mL}$ from the use in nanoparticle hemolysis test. The optimal time of sample incubation was 3 hours, and there was no significant difference in test results when rotation was used instead of mixing every 30 minutes (data not shown).

\subsection{Nanoparticle interference with the assay and approaches to overcome it}

The protocol described herein relies on the use of human blood anti-coagulated with Lithiumheparin. Other anti-coagulants are available commercially and may be used for this assay ${ }^{26}$. The same applies to the assay buffer. All nanoparticles in this study were suspended and diluted in calcium and magnesium free PBS. If a test nanomaterial is prone to agglomeration in PBS, other erythrocyte-friendly buffers, e.g. saline, may be used instead. We used this protocol for analysis of a variety of nanoparticles: nanoliposomes, PAMAM dendrimers, triazine dendrimers, nanoemulsions, water-soluble fullerene derivatives, and polystyrene nanoparticles, and repeatedly observed nanoparticle interference with the assay. Several examples of this interference are summarized below.

Generation 6 (G6) amine-terminated PAMAM dendrimers tested in this assay at concentration of $125 \mu \mathrm{g} / \mathrm{mL}$ resulted in $\sim 5 \%$ hemolysis (data not shown). This was consistent with previous studies of various nanomaterials demonstrating that cationic particles are more "reactive" than anionic or neutral particles and can damage cells (reviewed in ref. ${ }^{1}$ and ${ }^{7}$ ). When G5 amine terminated dendrimers were included in the analysis, no plasma-free hemoglobin was detected in supernatants (Figure 2A). When samples were analyzed during various steps of incubation, rapid (within first 15 minutes) coagulation of blood was observed (Figure 2B). Erythrocytes in the clot were protected from hemolysis, and blood clots were removed from the supernatants by centrifugation, giving a false-negative result. These findings were supported by a platelet aggregation study, in which G5 amine-terminated dendrimers caused approximately $80 \%$ platelet aggregation (Figure 2C). This data demonstrates that visual sample examination during all assay steps is important for the accurate interpretation of test-results. Another example of nanoparticle interference was observed when polystyrene nanoparticles were tested. Figure 2D shows the results of our assay for determination of the hemolytic properties of polystyrene nanoparticles. In this commonly used protocol, 20 and $50 \mathrm{~nm}$ polysterene nanoparticle size standards were incubated in whole blood, the blood was centrifuged to remove undamaged erythrocytes and nanoparticles, and the percent hemolysis was determined by colorimetric detection of hemoglobin in the supernatant. Under these conditions, untreated (i.e. commercially supplied) particles with 20 and 50nm diameters were strongly hemolytic. In the case of the 50nm particles, spectroscopic analysis indicated a reduction in hemolysis following dialysis. Visual inspection of the microcentrifuge tubes (Figure 2E), however, showed the dialyzed $50 \mathrm{~nm}$ particles adsorb hemoglobin (compared to control tube), and the adsorbed hemoglobin precipitates with the particles upon centrifugation-yielding a false negative result. This phenomenon was not observed with polystyrene nanoparticle less than 50nm in size, but was evident for larger, $80 \mathrm{~nm}$ polystyrene nanoparticles.

The most common mechanism of interference is due to the nanoparticle absorbance at or close to the assay wavelength $(540 \mathrm{~nm})$. This can be seen from Figure $3 \mathrm{~A}$, which shows the UV-Vis spectra of several nanoparticle samples. The third column of Figure 3B shows that the fullerene derivative, C3, causes hemolysis of almost 20\%. However, when the same nanoparticles were used in a control sample without blood (i.e. sample containing all assay components except 
the blood is substituted with PBS), the absorbance of this sample at $540 \mathrm{~nm}$ was also very high and when extrapolated against the hemoglobin standard curve corresponded to a percent hemolysis of $18 \%$ (Figure $3 \mathrm{~B} 4^{\text {th }}$ column). The same was true for citrated gold nanoparticles, some nanoemulsions, fullerene derivatives, and doxorubicin-loaded particles, all of which absorb near $540 \mathrm{~nm}$ (Figure 3A). For heavy particles (e.g. gold nanoparticles), an extra centrifugation step was used for removing the particles from the supernatant prior to evaluation on the plate reader. However, for the majority of nanoparticles, removal by centrifugation was not possible due to their small size and high solubility. For these particles, the results of the hemoglobin assay can be adjusted by subtraction of the absorbance of the no-blood control (i.e. sample containing all assay components except the blood is substituted by PBS). For example, for the $\mathrm{C} 3$ fullerene derivative, this adjusted result corresponds to a much lower percentage hemolysis (Figure 3B last column). If the degree of interference is very high (i.e. OD value of nanoparticle sample is above that of the highest calibrator in the hemoglobin standard curve), then dilution of the nanoparticle sample is required prior to test in order to obtain accurate results after the adjustment procedure.

\subsection{Relevance of in vitro assay to the in vivo testing}

To evaluate the relevance of the described in vitro assay for analysis of nanoparticle hemolytic properties, we have tested three nanotechnology-derived formulations approved by the US FDA for use in clinical applications. This analysis included, Doxil® (a liposomal formulation of doxorubicin), Abraxane $®$ (albumin bound Paclitaxel nanoparticles) and, Propofol®. (a nanoemulsion-based anesthetic agent). Doxil ${ }^{\circledR}$ and Propofol $®$ interfered with the assay when used at high concentrations. The results shown in Figure 4A and $4 \mathrm{~B}$ are adjusted to account for this interference. A low percentage hemolysis was observed with both formulations in vitro and it appeared to have no or weak relationship to the concentration of nanoparticle. This is in agreement with studies reporting low levels of erythrocyte damage by Doxil® in vivo, and Propofol ${ }^{\circledR}$ in vitro ${ }^{32-35}$. In both cases it was dose dependent, and minimized by using lower doses of formulations $32-35$. It is important to notice that when both Doxil and Propofol were analyzed at high concentration (labeled as "stock" in Figure 3A and 3B), the OD value of nanoparticle only control was above that of the assay highest calibrator. Subtraction procedure applied to adjust test result for the interference as described in the section above may not be accurate due to the high degree of nanoparticle interference with the assay. According to several studies in vitro percent hemolysis is rated as "no concern' when it varies from 5 to $25 \%$ 36-39. Our test results with Doxil® and Propofol® meet the criteria of some of these studies ${ }^{36}$. Abraxane ${ }^{\circledR}$ did not induce any damage to red blood cells when tested in vitro in our assay (Figure 4C), and there is no report in the literature on the hemolytic activity of this drug in vivo.

Thus, the assay described in our study demonstrated comparable negative test results for nanotechnology derived formulations tested in vivo. Although more comprehensive study is required to prove in vivo relevance of this in vitro method, the described assay can be used to exclude potentially harmful formulations from early preclinical testing (if in vitro result is $>50 \%$ hemolysis) and to suggest potential complications to monitor during in vivo studies of nanomaterials.

\section{Conclusions}

Many studies reported nanoparticle hemolytic propeties $10-25$. The majority of these studies utilized a spectrophotometric detection of plasma-free hemoglobin, and none of them reported specific controls to rule out nanoparticle interference. When it comes to preclinical safety testing of nanomaterials, ruling out false-positive or false-negative results is critical for accurate interpretation of data. Our study provides the first comprehensive insight to potential 
sources of this interference, demonstrates the usefulness of including "nanoparticle only" controls, and illustrates the importance of physico-chemical characterization of nanoparticle formulations and visually monitoring test samples to avoid false-positive or false-negative results.

\section{Supplementary Material}

Refer to Web version on PubMed Central for supplementary material.

\section{Acknowledgements}

This project has been funded in whole or in part with federal funds from the National Cancer Institute, National Institutes of Health, under contract N01-CO-12400. The content of this publication does not necessarily reflect the views or policies of the Department of Health and Human Services, nor does mention of trade names, commercial products, or organizations imply endorsement by the U.S. Government. We are grateful to Drs. Mansoor Amiji, Mark Kester, Eric Simanek and C60 Inc. for providing various nanoparticle formulations.

\section{References}

1. McNeil SE. J Leukoc Biol 2005;78(3):585-94. [PubMed: 15923216]

2. Ferrari M, Downing G. BioDrugs 2005;19(4):203-10. [PubMed: 16128604]

3. http://www.fda.gov/nanotechnology/

4. http://ncl.cancer.gov

5. http://www.astm.org (technical committee E56)

6. http://www.iso.org(technical committee TC229)

7. Brownlie A, Uchegbu IF, Schatzlein AG. Int J Pharm 2004;274(12):41-52. [PubMed: 15072781]

8. Cheng FY, Su CH, Yang YS, Yeh CS, Tsai CY, Wu CL, Wu MT, Shieh DB. Biomaterials 2005;26 (7):729-38. [PubMed: 15350777]

9. Chouly C, Bordenave L, Bareille R, Guerin V, Baquey A, Pouliquen D, Baquey C, Jallet P. Clin Mater 1994;15(4):293-301. [PubMed: 10147174]

10. Duguid JG, Li C, Shi M, Logan MJ, Alila H, Rolland A, Tomlinson E, Sparrow JT, Smith LC. Biophys J 1998;74(6):2802-14. [PubMed: 9635734]

11. Dutta T, Agashe HB, Garg M, Balasubramanium P, Kabra M, Jain NK. J Drug Target 2007;15(1): 89-98. [PubMed: 17365278]

12. Foger F, Noonpakdee W, Loretz B, Joojuntr S, Salvenmoser W, Thaler M, Bernkop-Schnurch A. Int J Pharm 2006;319(12):139-46. [PubMed: 16713146]

13. Goodman CM, McCusker CD, Yilmaz T, Rotello VM. Bioconjug Chem 2004;15(4):897-900. [PubMed: 15264879]

14. Guowei D, Adriane K, Chen X, Jie C, Yinfeng L. Int J Pharm 2007;328(1):78-85. [PubMed: 17014976]

15. Jin Y, Tong L, Ai P, Li M, Hou X. Int J Pharm 2006;309(12):199-207. [PubMed: 16377106]

16. Kainthan RK, Gnanamani M, Ganguli M, Ghosh T, Brooks DE, Maiti S, Kizhakkedathu JN. Biomaterials 2006;27(31):5377-90. [PubMed: 16854460]

17. Kim D, El-Shall H, Dennis D, Morey T. Colloids Surf B Biointerfaces 2005;40(2):83-91. [PubMed: 15642458]

18. Koziara JM, Oh JJ, Akers WS, Ferraris SP, Mumper RJ. Pharm Res 2005;22(11):1821-8. [PubMed: 16132346]

19. Lim SJ, Lee MK, Kim CK. J Control Release 2004;100(1):53-61. [PubMed: 15491810]

20. Nimesh S, Goyal A, Pawar V, Jayaraman S, Kumar P, Chandra R, Singh Y, Gupta KC. J Control Release 2006;110(2):457-68. [PubMed: 16325952]

21. Puzyr AP, Neshumaev DA, Tarskikh SV, Makarskaia GV, Dolmatov V, Bondar VS. Biofizika 2005;50(1):101-6. [PubMed: 15759508] 
22. Schubert MA, Muller-Goymann CC. Eur J Pharm Biopharm 2005;61(12):77-86. [PubMed: 16011893]

23. Verma AK, Sachin K, Saxena A, Bohidar HB. Curr Pharm Biotechnol 2005;6(2):121-30. [PubMed: $15853691]$

24. Vittaz M, Bazile D, Spenlehauer G, Verrecchia T, Veillard M, Puisieux F, Labarre D. Biomaterials 1996;17(16):1575-81. [PubMed: 8842361]

25. Zobel HP, Stieneker F, Atmaca-Abdel Aziz S, Gilbert M, Werner D, Noe CR, Kreuter J, Zimmer A. Eur J Pharm Biopharm 1999;48(1):1-12. [PubMed: 10477322]

26. ASTM International. Standard practice for assessment of hemolytic properties of materials. 2000

27. DeSilva B, Smith W, Weiner R, Kelley M, Smolec J, Lee B, Khan M, Tacey R, Hill H, Celniker A. Pharm Res 2003;20(11):1885-900. [PubMed: 14661937]

28. http://ncl.cancer.gov/NCL_Method_ITA-1.pdf

29. www.fda.gov/cber/summaries/120600bio10.ppt

30. Smolec J, DeSilva B, Smith W, Weiner R, Kelly M, Lee B, Khan M, Tacey R, Hill H, Celniker A, Shah V, Bowsher R, Mire-Sluis A, Findlay JW, Saltarelli M, Quarmby V, Lansky D, Dillard R,

Ullmann M, Keller S, Karnes HT. Pharm Res 2005;22(9):1425-31. [PubMed: 16132353]

31. Dobrovolskaia MA, McNeil SE. Nature Nanotechnology. 2007in press

32. Lewin SN, Mutch DG, Whitcomb BP, Liapis H, Herzog TJ. Gynecol Oncol 2005;97(1):228-33. [PubMed: 15790464]

33. Rivera E, Valero V, Arun B, Royce M, Adinin R, Hoelzer K, Walters R, Wade JL 3rd, Pusztai L, Hortobagyi GN. J Clin Oncol 2003;21(17):3249-54. [PubMed: 12947059]

34. Chen H, Zhang Z, Almarsson O, Marier JF, Berkovitz D, Gardner CR. Pharm Res 2005;22(3):35661. [PubMed: 15835740]

35. Murphy PG, Davies MJ, Columb MO, Stratford N. Br J Anaesth 1996;76(4):536-43. [PubMed: 8652327]

36. Amin K, Dannenfelser RM. J Pharm Sci 2006;95(6):1173-6. [PubMed: 16639718]

37. Krzyzaniak JF, Raymond DM, Yalkowsky SH. PDA J Pharm Sci Technol 1996;50(4):223-6. [PubMed: 8810837]

38. Krzyzaniak JF, Alvarez Nunez FA, Raymond DM, Yalkowsky SH. J Pharm Sci 1997;86(11):12157. [PubMed: 9383728]

39. Krzyzaniak JF, Yalkowsky SH. PDA J Pharm Sci Technol 1998;52(2):66-9. [PubMed: 9610170] 


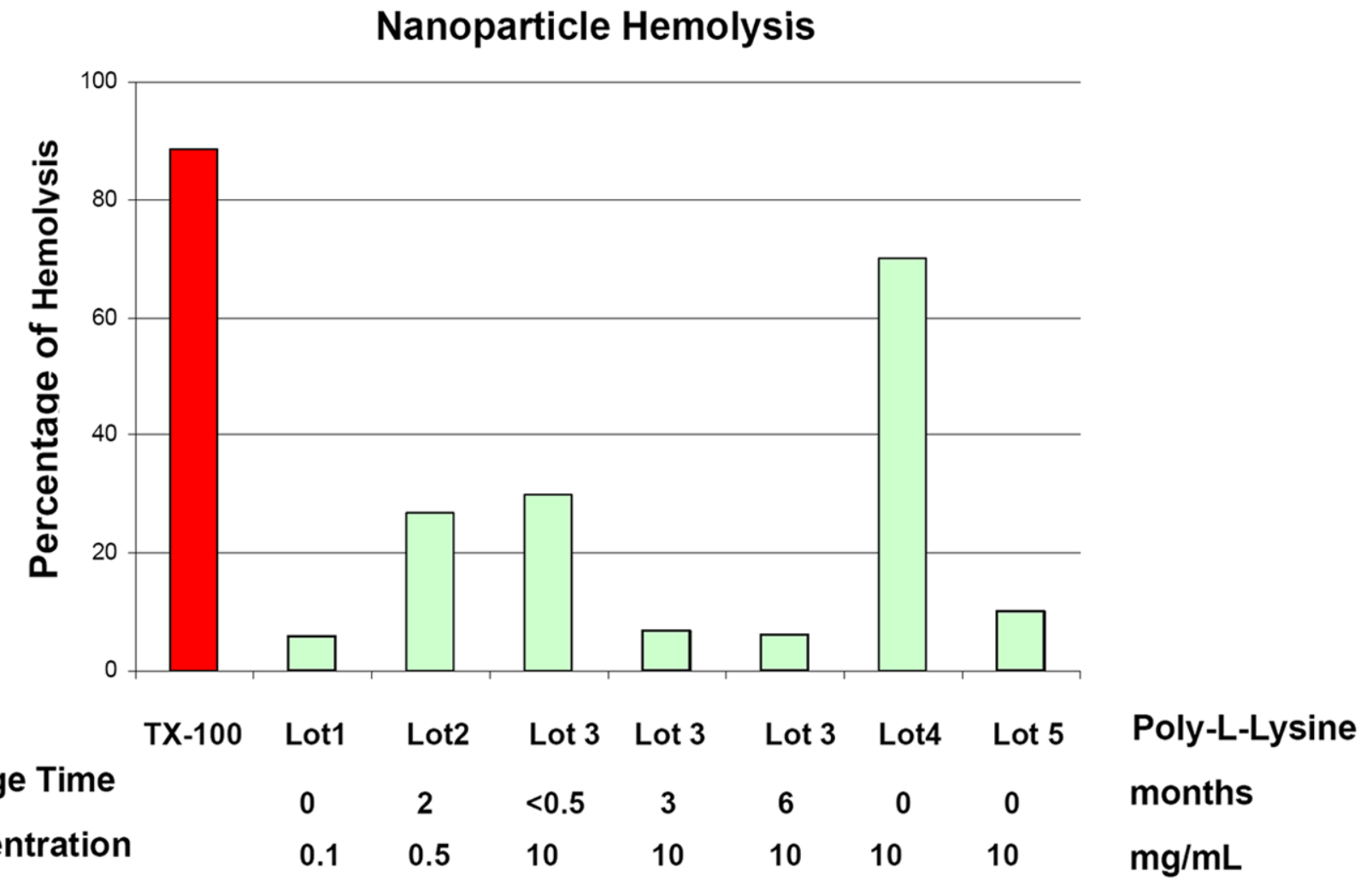

Figure 1. PLL disqualified as a positive control

Poly-L-Lysine (PLL) is cationic polymer. Evaluation of PLL in 96-well format assay revealed a high degree of inter-lot variability. Although precision and accuracy of PLL in pre-study validation conducted using the same lot of polymer met the acceptance limits (i.e. \% CV and PDFT $<15 \%$, Table $2 \mathrm{~A}$ ), the high degree of inter-lot variability disqualified this material from further use as positive control. Each bar represents mean of at least two duplicate responses. Percent CV for each duplicate was less than 10. 

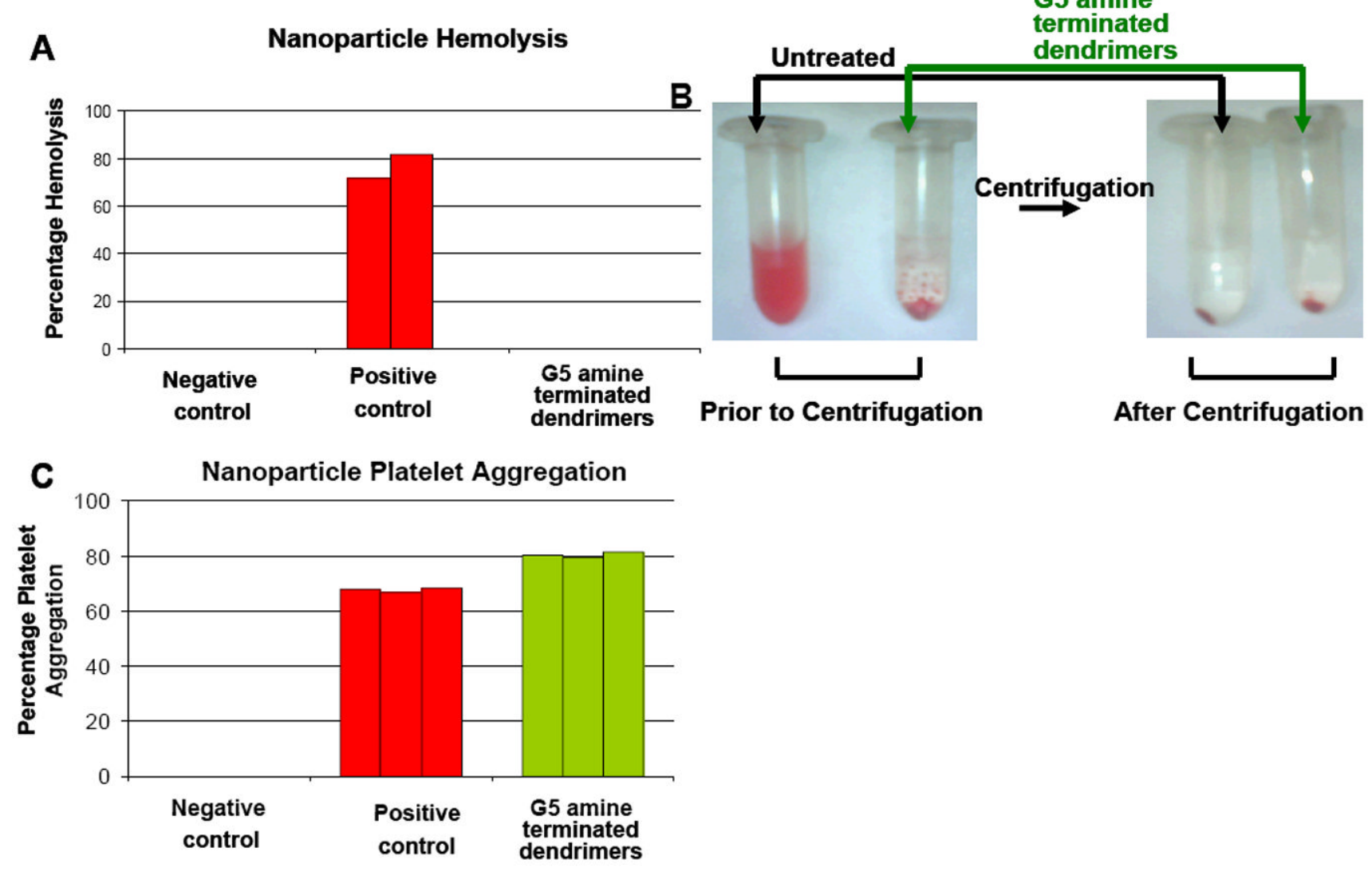

D

E
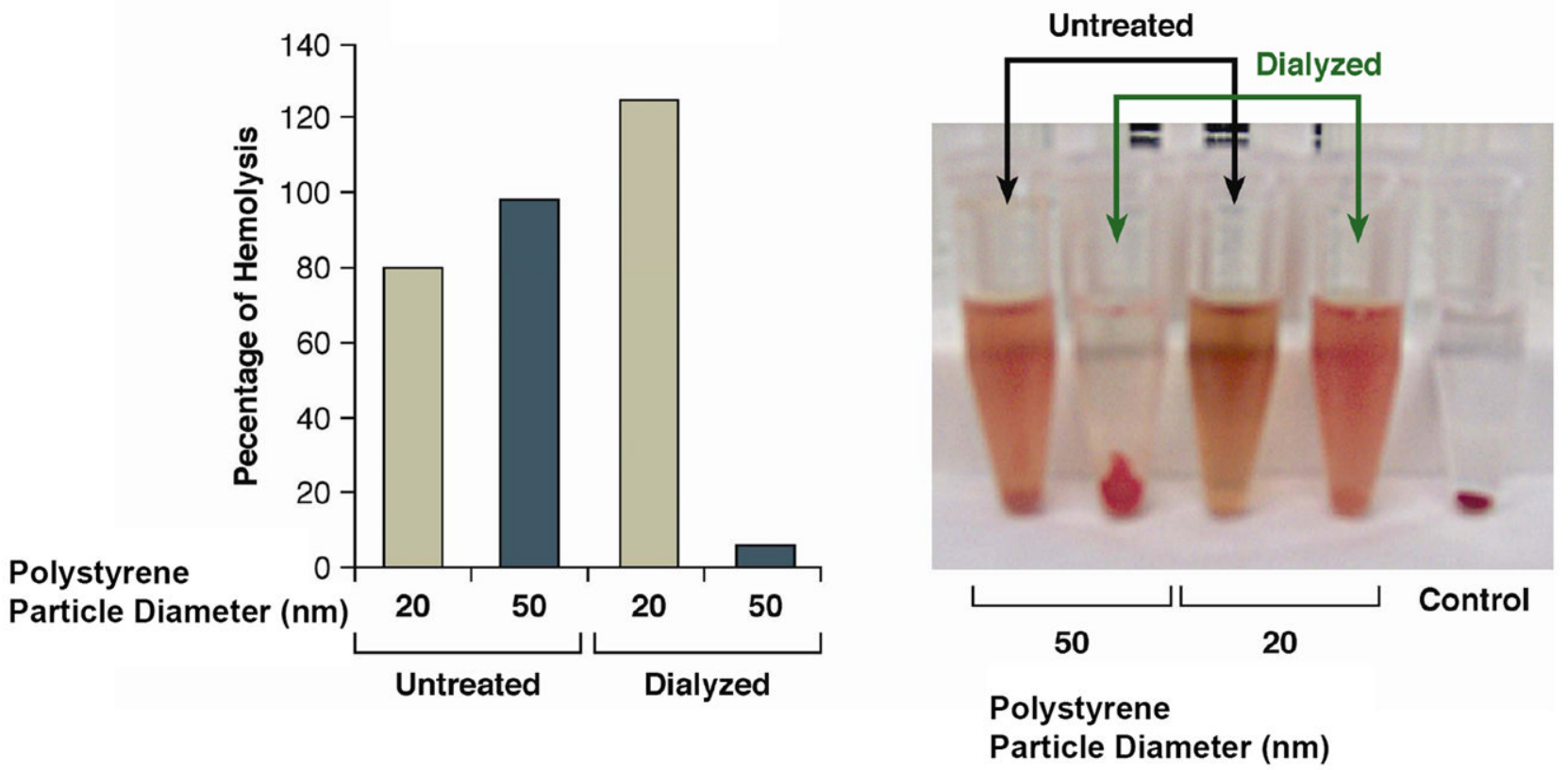

Figure 2. Nanoparticles interfere with the hemolysis assay

(A) G5 amine terminated dendrimers were analyzed in duplicate, and no plasma-free hemoglobin was detected in supernatants. Three tests were conducted for the dendrimers. Each 
bar represent mean of the duplicate response with \%CV less than 20. (B) The same samples were analyzed during various steps of incubation and rapid (within first 15 minutes) coagulation of blood was observed. Erythrocytes in the clot were protected from hemolysis, and blood clots were removed from the supernatants by centrifugation, giving a false-negative result. (C) The same samples were analyzed in platelet aggregation test. Each bar is mean of duplicate response with \%CV less than 20. Three tests were conducted. (D)-Analysis of 20 and $50 \mathrm{~nm}$ polysterene nanoparticles. Each bar represents mean of three test results. Each test results was obtained from a duplicate response with \% CV less than 20. (E) Visual inspection of the microcentrifuge tubes containing blood samples treated with 20 and $50 \mathrm{~nm}$ polysterene nanoparticles before and after dialysis. 
A

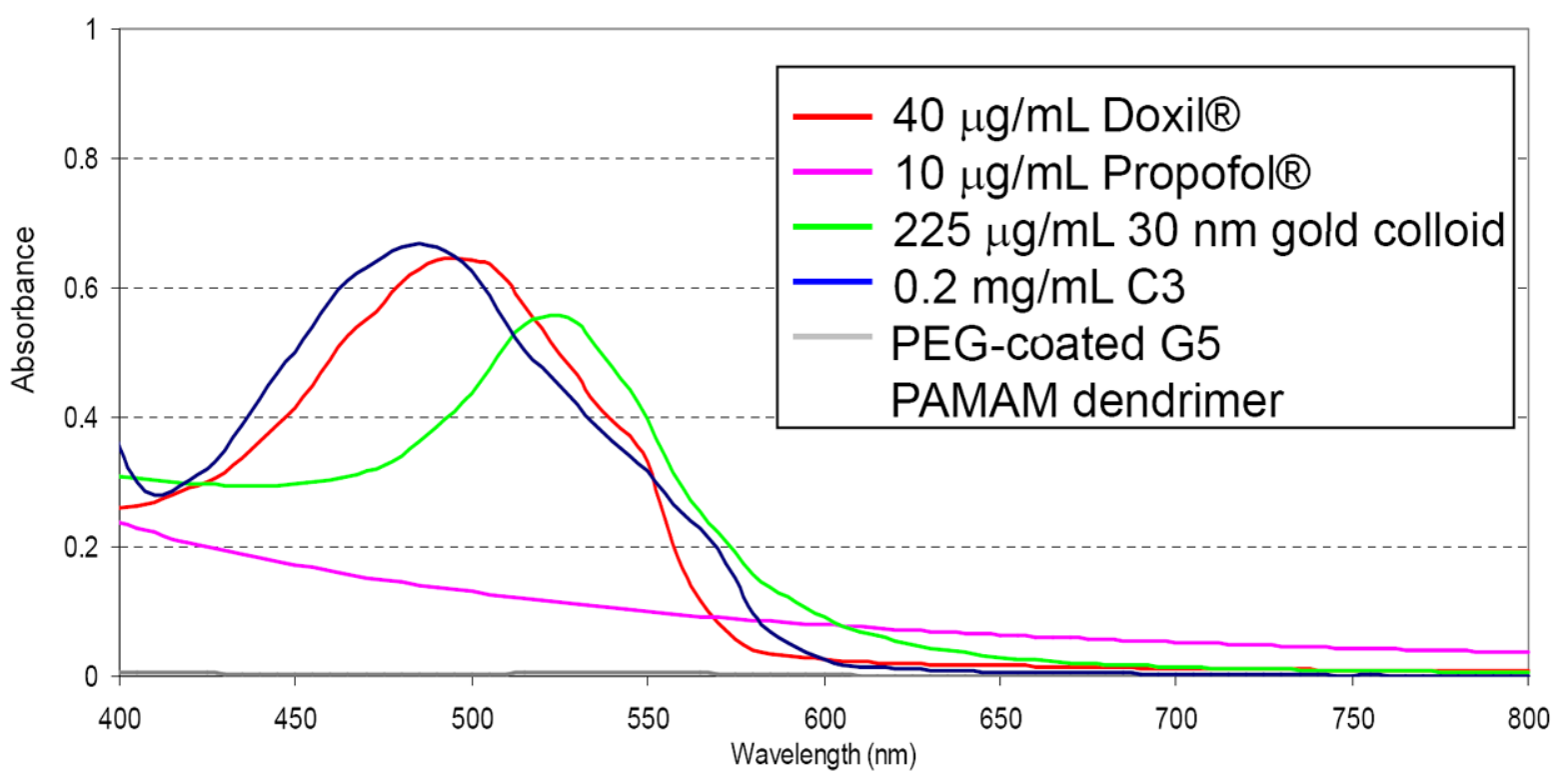

Nanoparticle Hemolysis

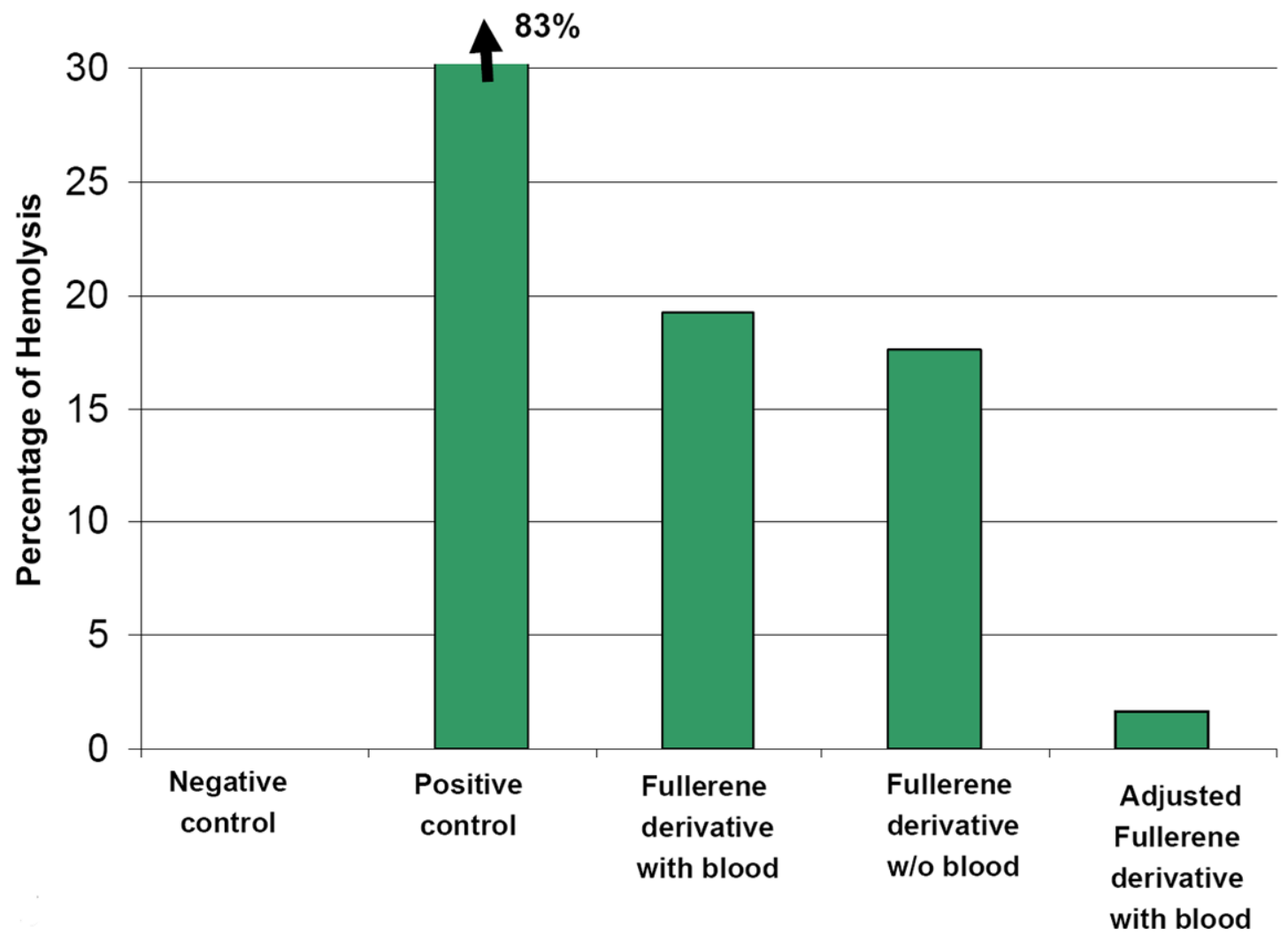

Figure 3. Nanoparticle optical properties as a source of interference with hemolysis assay 
A) UV-Vis spectrum of several nanoparticle samples in the vicinity of the assay detection wavelength of $540 \mathrm{~nm}$. Doxil@, the $30 \mathrm{~nm}$ gold colloid, and fullerene derivative C3 at concentrations shown in the figure all have significant absorbance near $540 \mathrm{~nm}$. UV-Vis spectra were recorded using a Molecular Devices SpectraMax M5 (Sunnyvale, CA). Samples were prepared in Milli-Q water and measured in quartz microcuvettes $(b=10 \mathrm{~mm}$, QS105.250, Hellma, Plainview, NY). Spectra were collected from $200-800 \mathrm{~nm}$ in $5 \mathrm{~nm}$ steps with Milli$\mathrm{Q}$ water as the reference. B) Analysis of fullerene derivative C3 in hemolysis assay. Results of one of three tests are shown. Each bar represents mean of a duplicate response with \%CV less than 15 

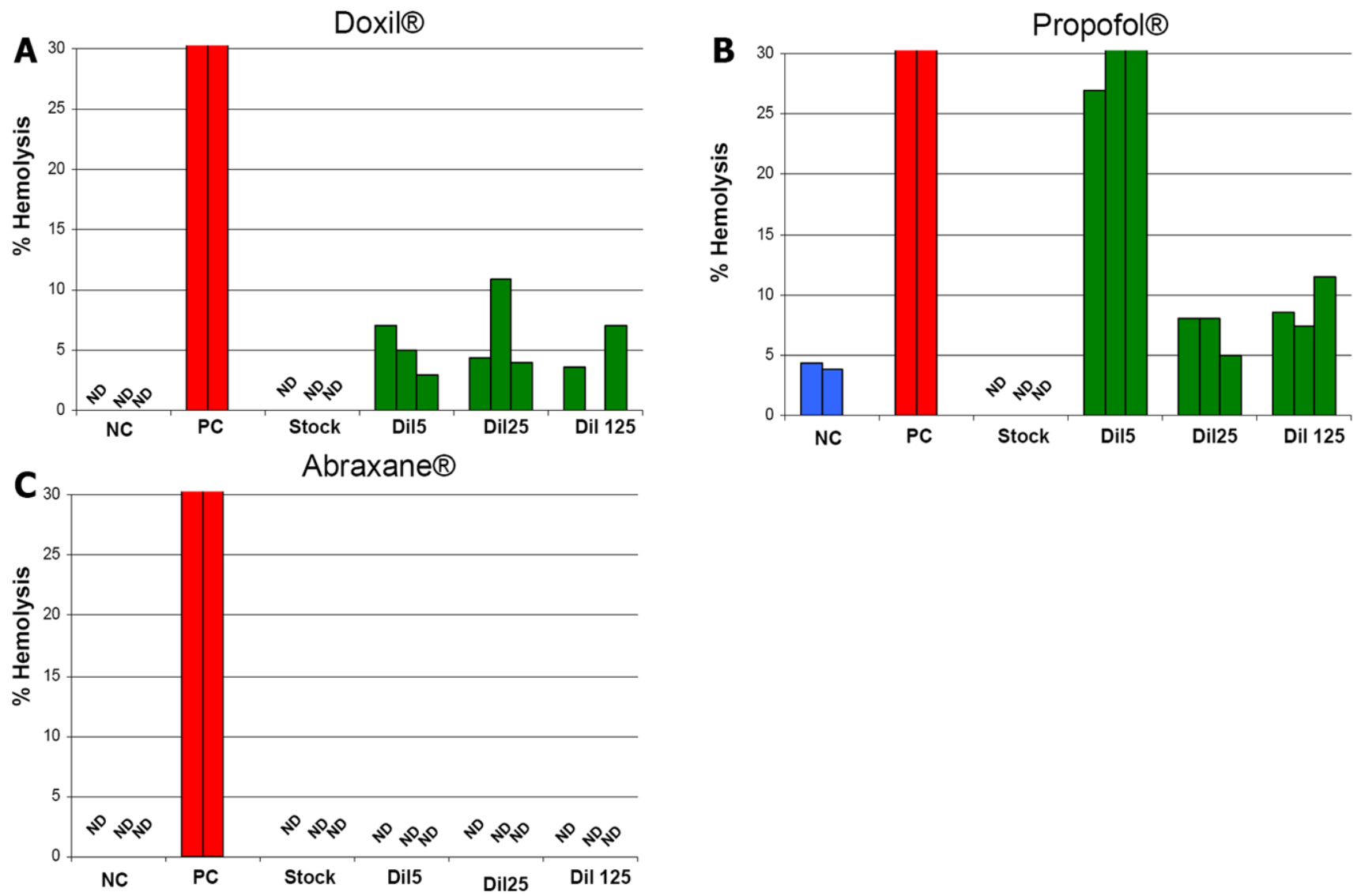

Figure 4. Hemolysis assay applied to nanoparticulate pharmaceuticals approved fro clinical use Hemolytic properties of commercially available nanoparticle formulations approved for clinical use Doxil@ (A), Propofol ${ }^{\circledR}(B)$ and Abraxane ${ }^{\circledR}(C)$ were studied in vitro. Three tests were conducted for each nanoparticle sample. Each bar represents mean of a duplicate response with \%CV less than 15 . Stock refers to nanoparticle formulations used directly from the commercially supplied vial. This stock was subjected to three serial 1 to 5 dilutions in PBS; these dilutions are labeled as Dil 5 (dilution of the stock 1 to 5), Dil 25 (dilution of the stock 1 to 25 ) and Dil 125 (dilution of the stock 1 to 125 ). NC is negative control (PBS). PC - is positive control (Triton X-100). The scale was reduced to focus on nanoparticle percent hemolysis. Hemolysis observed for positive control sample was above $80 \%$. ND refers to no detectable hemolysis. 


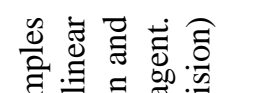

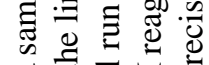

는

政记

a $:$.

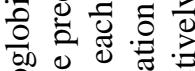

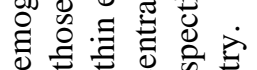

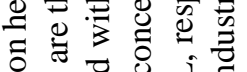

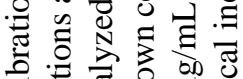

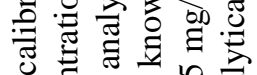

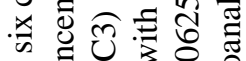

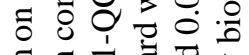

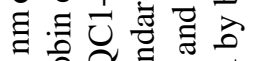

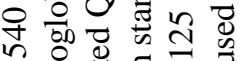

ธ

菏

言

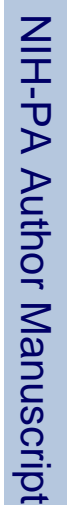

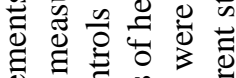

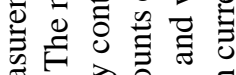

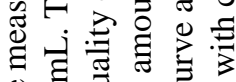
ช 들

然

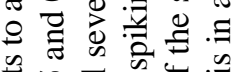

过

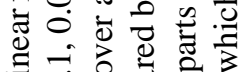

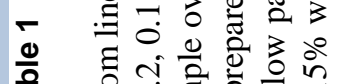

๙ 일

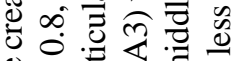
눙

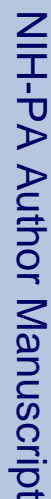

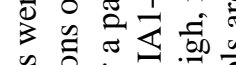

o 을

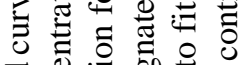
急

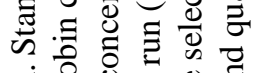
. 密

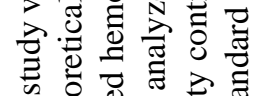



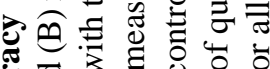

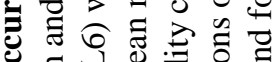
๘

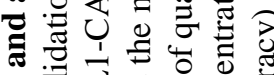
产安里 중

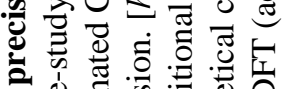

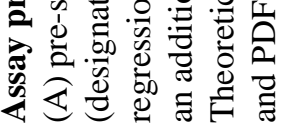

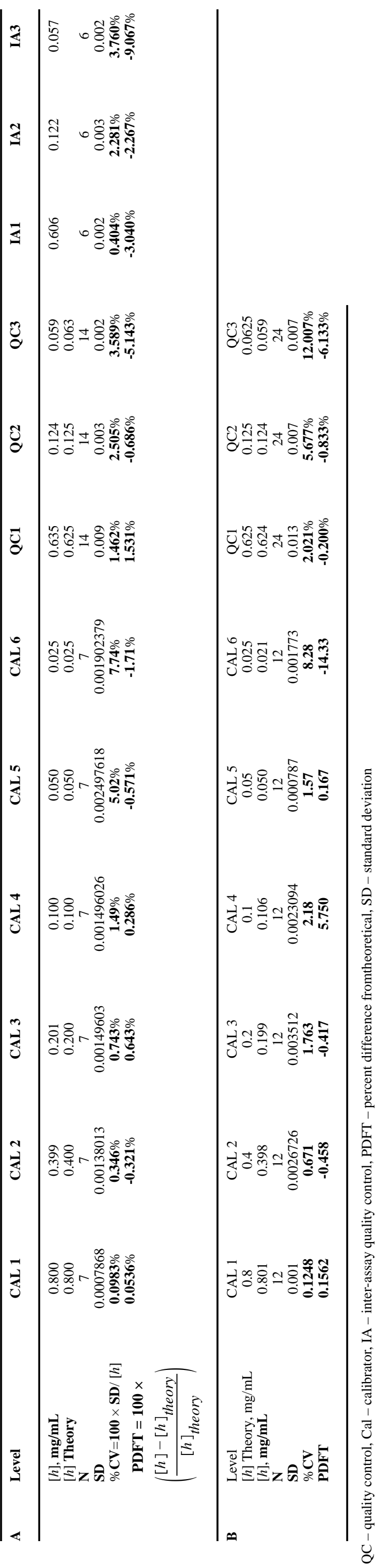




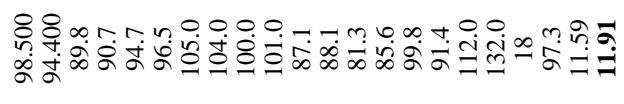

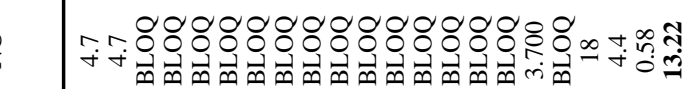

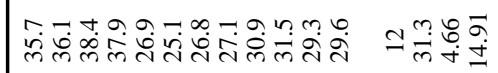

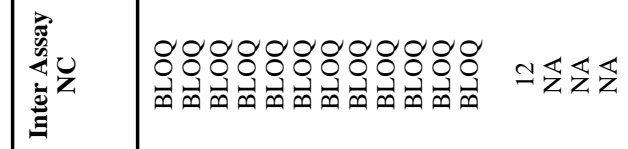

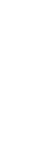

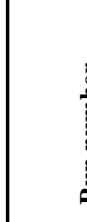

|-

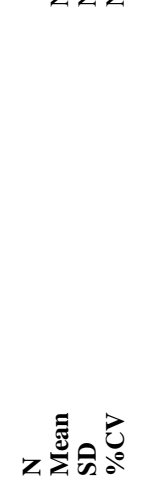

Nano Lett. Author manuscript; available in PMC 2009 August 1. 